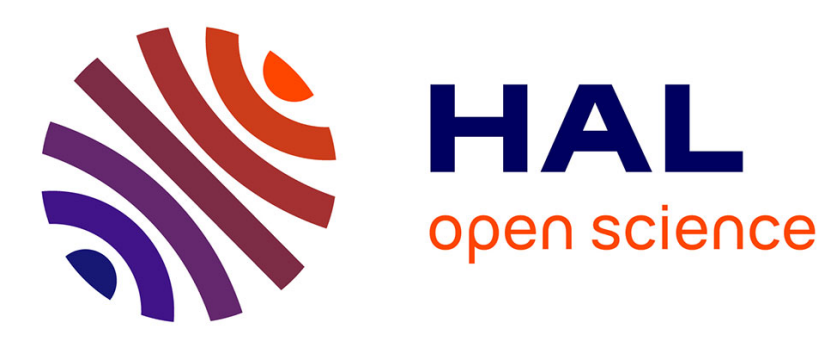

\title{
Under-frequency Load Shedding Schemes Characteristics and Performance Criteria
}

Barnabé Potel, Vincent Debusschere, Florent Cadoux, Leticia de Alvaro Garcia

\section{- To cite this version:}

Barnabé Potel, Vincent Debusschere, Florent Cadoux, Leticia de Alvaro Garcia. Under-frequency Load Shedding Schemes Characteristics and Performance Criteria. PowerTech 2017, Jun 2017, Manchester, United Kingdom. hal-01549263

\section{HAL Id: hal-01549263 \\ https://hal.science/hal-01549263}

Submitted on 4 Sep 2019

HAL is a multi-disciplinary open access archive for the deposit and dissemination of scientific research documents, whether they are published or not. The documents may come from teaching and research institutions in France or abroad, or from public or private research centers.
L'archive ouverte pluridisciplinaire HAL, est destinée au dépôt et à la diffusion de documents scientifiques de niveau recherche, publiés ou non, émanant des établissements d'enseignement et de recherche français ou étrangers, des laboratoires publics ou privés. 


\section{Under-frequency Load Shedding Schemes Characteristics and Performance Criteria}

\author{
Barnabé Potel, Vincent Debusschere \\ Univ. Grenoble Alpes, G2Elab, \\ F-38000 Grenoble, France \\ barnabe.potel@g2elab.grenoble-inp.fr \\ vincent.debusschere@g2elab.grendoble-inp.fr
}

\author{
Florent Cadoux \\ Fondation Partenariale de Grenoble INP \\ F-38000 Grenoble, France \\ florent.cadoux@g2elab.grenoble-inp.fr
}

\author{
Leticia de Alvaro Garcia \\ Enedis - France \\ leticia.de-alvaro@enedis.fr
}

\begin{abstract}
Our work focuses on the European Network Code on Emergency and Restoration (NC-ER) which introduced an harmonization requirement of Under-frequency Load Shedding (UFLS) schemes. This new requirement implies that in the medium term, the UFLS scheme of all European countries will have to evolve. It stipulates an acceptable range for the main factors that define an UFLS scheme, namely: the number of load shedding steps, the percentage of load shed in each step, and the accuracy of the frequency measurement that is implemented in the protection relays. In this context, the contribution of this paper is twofold: first, we define a new performance criterion for UFLS schemes; and second, we apply this criterion to two opposite archetypal schemes, in order to highlight the pros and cons of both approaches.
\end{abstract}

Index Terms-Under-frequency Load Shedding (UFLS), Defense plan, Power system, Stability

\section{INTRODUCTION}

An Under-frequency Load Shedding (UFLS) scheme is designed to prevent a power system from a black-out due to a large power imbalance. Most current used schemes in the European grid follow the following traditional design: a predefined amount of load is shed automatically whenever frequency decreases below certain predefined thresholds [1].

The Network Code on Emergency and Restoration (NC-ER) [2] is a legally binding document which compels ENTSO-E members to update their UFLS schemes in order to harmonize technical operations in the European power grid. When a large grid event which involves load shedding occurs, a balanced participation of each country seems to be the fairest and safest response. As a general rule, UFLS schemes that are currently implemented by European TSOs, and that were inherited from the past, do not comply with the latest NC-ER and will have to be updated.

Two archetypal examples of UFLS schemes are the following. In what could be called the "current" scheme, only a few UFLS steps are used by each TSO, the associated frequency threshold being different from one TSO to another, the amount of load shed in each step is large and frequency measurement is relatively inaccurate due to the use of protective relays designed decades ago. Conversely, in what could be called the "future" situation, many UFLS steps containing only relatively

The work reported in the paper has been developed in the framework of the Enedis Industrial Chair On Smart Grids research program. little load would be used, and the accuracy of frequency measurement would be improved. When implementing the NC-ER, national TSOs will have to choose the parameters of their UFLS scheme inside the allowed range and to make sure that the new settings actually improve system stability with respect to the current situation; this raises the question of evaluating the performance of a given UFLS scheme.

As an example, "Scheme A", defined by Table I, illustrates the type of scheme that is currently used by TSOs. It is composed of four UFLS steps. Such a traditional scheme typically allows a relatively large inaccuracy in frequency measurement; hence this scheme is not compliant with the guidelines described in the NC-ER (the new UFLS scheme shall be designed with six steps between 48 and $49 \mathrm{~Hz}$, each step should shed between 5 and $10 \%$ for a total of $45 \%$ shed load). In that context, the objective of this work is to compare the performance of a typical current UFLS scheme with the performance of a newer NC-ER-compliant scheme. To this end, we will first introduce a new performance metric for UFLS schemes, and then apply this metric to perform our comparison.

A prospect of this UFLS design, not studied in this paper, is the future challenge due to the steady increase of distributed generation: in this context, disconnecting an entire MV feeder (both load and generation at once) by triggering an UFLS relays in the primary substation may prove too brutal. As a consequence, new solutions will probably be required in order to preserve the efficiency of the load shedding mechanism [3][6].

\section{UFLS MECHANISM}

\section{A. Principle}

Different kinds of events may cause the frequency to drop to the point where automatic load shedding triggers. For example, large generators may trip, or transmission lines may disconnect leading to the grid separation into subareas with potentially large power imbalances. Then frequency decreases according to the swing equation (1). The primary reserve of frequency regulation may not be sufficient to contain the frequency drop in such a severe situation; whenever power consumption exceeds power generation so much that the imbalance cannot be dealt with by quickly ramping up generators, some consumers 
must be immediately disconnected from the grid to contain the contingency. As a consequence, whenever frequency reaches the first threshold of the UFLS scheme (which is commonly set to $49 \mathrm{~Hz}$ ), a fraction of the consumption is shed. If frequency keeps dropping, additional load is shed at lower frequency thresholds. Under-frequency load shedding is thus the last action to avoid an extensive black-out.

\section{B. UFLS scheme design}

The steps of an UFLS Scheme are defined by their frequency threshold and the percentage of load to shed. After enough load is shed, frequency eventually goes up and the frequency drop is contained. The amount of load shed should thus be sufficient, but not excessive, since an overly aggressive UFLS scheme would actually be counter-productive. Indeed, if too much load is shed, the under-frequency situation may be followed by an over-frequency situation that could lead to the disconnection of generators [7]. In such a case, the UFLS mechanism would overshoot and actually worsen the power generation deficit.

Fig. 1 shows an example. The simulation was based on equations that will be presented below in Section III. An imbalance of $20 \%$ between generation and consumption is simulated and three schemes triggering at $49 \mathrm{~Hz}$ are compared. The first one is shedding $19 \%$ of the consumption, the second one $20 \%$ and the last one $22 \%$. The final value of frequency is reported in the legend of the graph on Fig. 1; this value may be determined by a longer simulation or simply by using equations (2) and (3) below.

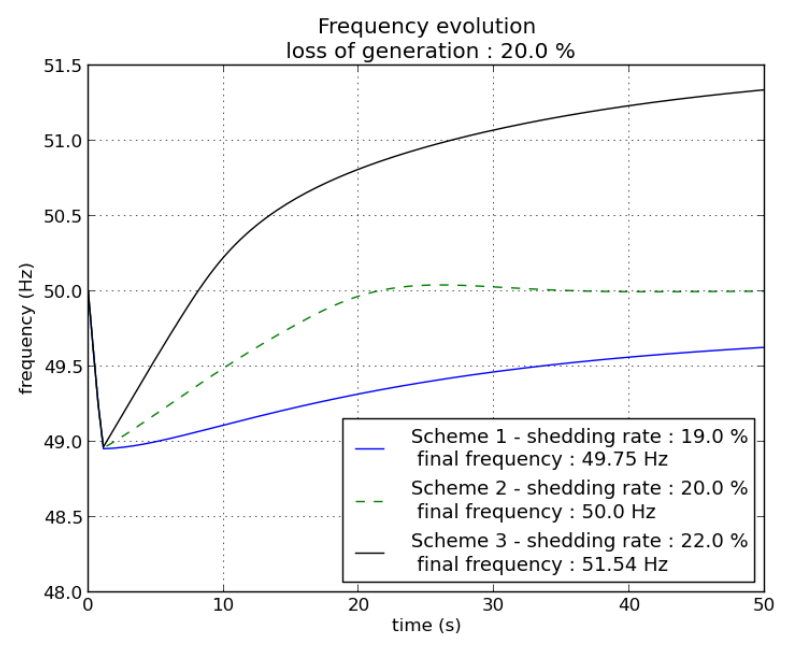

Fig. 1. Evolution of frequency for different percentages of load shed.

Fig. 1 demonstrates that our three schemes exhibit very different behaviors.

- Scheme 3 is shedding too much load and leads to an over-frequency situation. The most sensitive generators may disconnect.

- Scheme 1 simply contains the frequency drop and the final frequency is lower than its initial value.
- Scheme 2 not only contains the frequency drop, but also drives frequency back to its initial value. Nevertheless, a small temporary overshoot in frequency may be observed; this is due to the frequency regulation.

In section IV-B below, we will elaborate on this example to devise our performance criterion for UFLS schemes.

TABLE I

SCHEME A

\begin{tabular}{ccccc}
\hline Frequency threshold & $49 \mathrm{~Hz}$ & $48.5 \mathrm{~Hz}$ & $48 \mathrm{~Hz}$ & $47.5 \mathrm{~Hz}$ \\
\hline Percentage of load shed $^{a}$ & $15 \%$ & $15 \%$ & $15 \%$ & $15 \%$ \\
\hline Total of load shed & $15 \%$ & $30 \%$ & $45 \%$ & $60 \%$ \\
\hline
\end{tabular}

${ }^{a}$ This percentage is taken from national load.

By contrast with the UFLS Scheme A, Table II shows one of the many possible schemes that comply with the NC-ER. Note that this scheme contains six steps, two more than Scheme A.

TABLE II

SCHEME B

\begin{tabular}{lllllll}
\hline Frequency threshold & $49 \mathrm{~Hz}$ & $48.8 \mathrm{~Hz}$ & $48.6 \mathrm{~Hz}$ & $48.4 \mathrm{~Hz}$ & $48.2 \mathrm{~Hz}$ & $48 \mathrm{~Hz}$ \\
\hline $\begin{array}{l}\text { Percentage of load } \\
\text { shed }{ }^{a}\end{array}$ & $5 \%$ & $8 \%$ & $8 \%$ & $8 \%$ & $8 \%$ & $8 \%$ \\
\hline Total of load shed & $5 \%$ & $13 \%$ & $21 \%$ & $29 \%$ & $37 \%$ & $45 \%$ \\
\hline
\end{tabular}

${ }^{a}$ This percentage is taken from the national load.

\section{POWER GRID MODELING FOR UFLS STUDIES}

\section{A. Grid model}

The grid is modeled by a single synchronous machine which provides the generation, inertia and primary frequency regulation. The secondary frequency regulation is not taken into account because its time constant is too large to be considered.

Swing equation: the frequency evolution is ruled by the swing equation:

$$
\frac{d \bar{f}}{d t}=\frac{\Delta \bar{p}}{2 H}
$$

$\bar{f} \quad$ frequency in p.u.

$\Delta \bar{p} \quad$ generation and consumption difference in p.u.

$H \quad$ inertia

Primary frequency regulation: The frequency regulation is represented by a proportional gain $g_{d}$ and saturates at $49.8 \mathrm{~Hz}$ and $50.2 \mathrm{~Hz}$ [8], followed by a first-order low-pass filter with time constant $\tau$. The symbol $s$ denotes Laplace-transform.

$$
g=g_{0}\left(1+g_{d}(1-\bar{f})\right) \frac{1}{(1+\tau s)}
$$

$g_{0} \quad$ initial generation (after triggering event)

$g_{d}$ generation droop

$\tau$ time constant for generator response 
Consumption: We modeled the load as depending linearly from frequency [9].

$$
p=p_{0}\left(1+k_{p f}(\bar{f}-1)\right)
$$

$p_{0} \quad$ initial consumption considering the load shedding

$k_{p f}$ frequency-sensitivity of consumption

Simulations were conducted using the following values:

$\begin{array}{ll}H & 6 \text { seconds } \\ g_{d} & 5 \% / \mathrm{Hz} \\ k_{p f} & 1 \% / \mathrm{Hz}\end{array}$

A sensitivity study with respect to these parameters is provided in the next section.

\section{B. Relays}

On November 4th, 2006, an incident led to triggering the first step of the UFLS scheme in several European countries. It revealed that a part of the relays contained in the first step did not trigger because of frequency measurement inaccuracy [10].

This behavior is added in our model, considering that the load shed at a step follows a normal distribution depending on the frequency. The standard deviation of this distribution is directly linked to the frequency measurement accuracy. The tripping delay of our "inaccurate relays" is considered as depending on the accumulated time spent in the vicinity of the frequency threshold: the longer frequency remains at a certain value (frequency threshold \pm 3 standard deviation) the more relays trigger. See Fig. 2 for an example of this behavior.

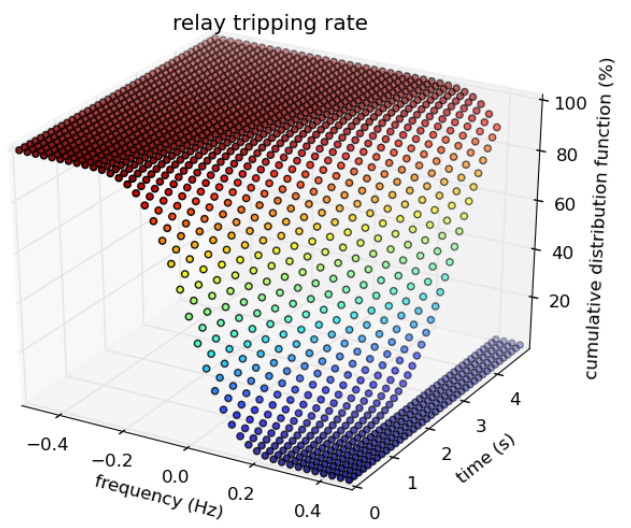

Fig. 2. Percentage of triggered relays of a step for a frequency threshold at $0 \mathrm{~Hz}$ and a standard deviation of $0.1 \mathrm{~Hz}$

\section{Premature loss of generation caused by low frequency}

During an under-frequency situation, generator tripping is generally assumed to begin at $47.5 \mathrm{~Hz}$. In fact, the event that occurred on November 4th, 2006 in Europe and the countrywide black-out that occurred on September 28th, 2003 in Italy showed that generators may disconnect before frequency actually reaches the $47.5 \mathrm{~Hz}$ limit, and sometimes even before it reaches $49 \mathrm{~Hz}$. We do not implement these situations explicitly in our simulation, since they may simply be considered as a larger initial loss of generation.

Moreover, most of the generators which tripped during the event of 2006 were meeting grid requirements (tripping at those frequencies was their expected response); but these requirements have evolved since then, and generators, nowadays, should not trip before reaching $47.5 \mathrm{~Hz}$. Nevertheless, the physical design of generators (that depends on choices made by their manufacturers) may be such that the actual range of frequency supported by the machine is smaller than the range of frequency implemented in the protection relays (which depends on grid requirements). Thus the possibility of losing generators before reaching $47.5 \mathrm{~Hz}$ or $51 \mathrm{~Hz}$ may still exist.

\section{COMPARISON OF VARIOUS UFLS SCHEMES}

A. Robustness of an UFLS scheme with respect to the triggering event

As developed in the introduction, under-frequency situations may be caused by various contingencies. In particular, they may arise due to the loss of transmission lines causing the network to split into subareas. It is important to notice that the parameters of these sub-networks cannot be determined in advance; hence, no assumption should be made regarding (A) the amount of imbalance that the UFLS scheme will have to deal with, nor (B) the characteristics (such as inertia) of the network after the event, nor $(\mathrm{C})$ the location of the resources (such as MV-feeders or entire HV/MV substations) that the UFLS will be able to rely on.

The last point $(\mathrm{C})$ leads to the relatively straightforward conclusion that UFLS resources should be spread relatively evenly in the network. It would probably not be a good idea, for instance, to locate all the resources of the first UFLS step in the North of a country, and all the resources of the second UFLS step in the South.

The first and second points (A) and (B) are however less obvious. Ensuring that an UFLS scheme will be robust with respect to the total initial imbalance and the system inertia calls for detailed analysis, which will be the object of the following sections.

\section{B. Performance criterion}

The performance metric used by ENTSO-E to support the new requirements is [11] the steady-state value reached by the frequency after the load-shedding : the scheme is considered as efficient if this value is close to the initial value of $50 \mathrm{~Hz}$ for 8 different imbalances $(1,5,10,20,30,40,50$ and $60 \%)$.

Taking a different perspective at the matter, we argue that it would also be reasonable to measure the performance of an UFLS scheme by the maximum frequency which can be reached after load shedding, for any imbalance lower than the 
total amount of load in the UFLS scheme. By comparison with the performance criterion of ENTSO-E [11], this means two things:

- first, we are checking the value of frequency for the whole range of possible imbalances, and not only for a few predefined values of the imbalance.

- Second, we are checking the value of frequency at any time after load shedding occurs, not only its steady-state value.

The rationale of our performance metric is the following: UFLS is an automatic and fast response to re-establish power balance, it should thus be judged by its ability to maintain system stability and not by its ability to return frequency to its nominal value. If the steady-state frequency value after load shedding stabilizes to a low (yet above $47.5 \mathrm{~Hz}$ ) value, this is not detrimental to the power system: the point is that a full black-out was avoided, and TSOs will then be able to take actions to get the frequency back to its initial value, by starting up additional generation units or manually shedding some more load if necessary. As a consequence, a given UFLS plan should not be penalized based on the fact that it leads to an under-frequency situation. On the contrary, over frequency situations should be avoided because generators as a whole will disconnect much faster in case of over-frequency. Avoiding over-frequency situations, at any time (not only in steady-state) and for any reasonable value of the initial imbalance, is thus the aim of our performance criterion.

The value of the maximum frequency after load shedding depends on relays characteristics and grid parameters such as inertia. If the grid splits into subareas, each subarea may have its own characteristics which are not predictable. We thus need to carry out a sensitivity study with respect to the three following important grid parameters: system inertia, droop constant and frequency-sensitivity of consumption.

The principle of our work is to time-simulate various imbalances and to plot the maximum value of frequency that is reached after load shedding for each scenario. Fig. 3 shows how the maximum frequency changes when loss of generation is increased. Two schemes are compared: one is representative of "current" schemes, and the other is representative of "future" schemes that will fulfill the NC-ER requirements (we used the schemes shown in Table I and II).

Curves start from $3 \%$ (as the primary reserve is around $1 \%$ and the consumption decrease is around $1 \%$ too for triggering the first UFLS step at $49 \mathrm{~Hz}$ ). These curves look like saw-teeth. Their number is directly linked to the number of steps for each scheme: six for Scheme B and four for Scheme A. It is clear that taking a limited numbers of imbalances does not allow to have an overview of the performances of an UFLS scheme.

Local minima are observed on Fig. 3 whenever the loss of generation reaches a value such that a new step of the UFLS scheme must be activated; in such a case, the maximum frequency after shedding (on the $\mathrm{y}$-axis) increases quickly with the amount of generation lost (on the x-axis). Indeed, losing only slightly more generation will lead the frequency to reach a slightly lower value and will potentially cause a

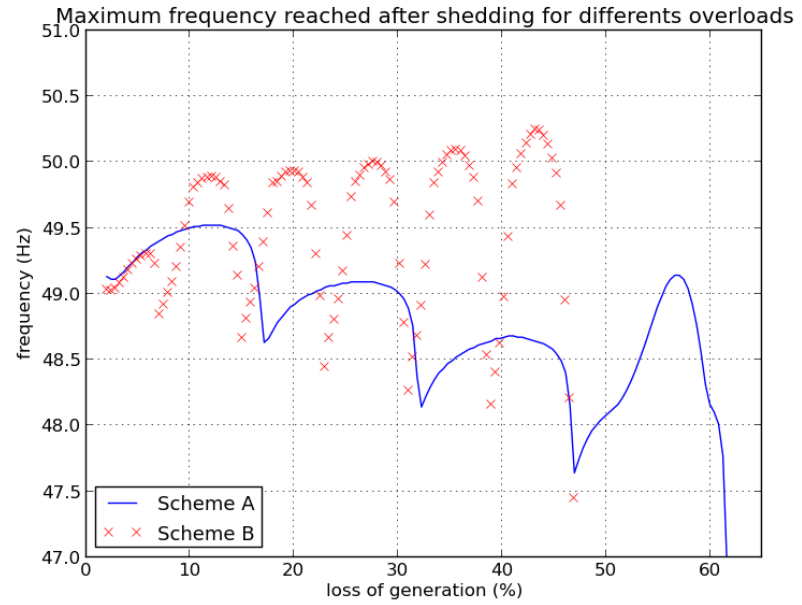

Fig. 3. Frequency progression for different shed percentages

large number of additional UFLS relays to trigger. In the extreme situation where all relays would trigger exactly at the same frequency, that is to say, if the frequency measurement inaccuracy was not taken into account, the curves depicted on Fig.3 would go up vertically after each local minimum; local maxima would be reached immediately after local minima; and whenever a minimum would be attained, one would read the frequency threshold of the corresponding UFLS step on the yaxis. However, because of frequency measurement inaccuracy, the slope of the curve after local minima is not vertical, local maxima are reached about halfway between two UFLS steps (not right after a step), and minima are reached for frequency values that are slightly higher than the theoretical frequency thresholds of the UFLS scheme.

Fig. 3 shows that the "current" scheme, where the thresholds of UFLS relays are distributed with a standard deviation of $50 \mathrm{mHz}$ [10], never exceeds the nominal frequency of $50 \mathrm{~Hz}$ : as a consequence, there is no risk of over-frequency situation. In comparison, the "future" scheme, where the standard deviation is only $10 \mathrm{mHz}$ (representing $30 \mathrm{mHz}$ precision according to [12]), drives frequency to values well above $50 \mathrm{~Hz}$. The newer scheme benefits from having more steps of smaller size than the "current" scheme; but the fact that frequency is measured more accurately favors the occurrence of over-frequency situations. The main differences between these schemes are shown in Table III.

The results of Table III may be generalized, but the curves from Fig. 3 tremendously depend on grid and relays parameters (inertia, accuracy of frequency measurements, etc). If those parameters vary, the curves will keep approximately the same shape but may be shifted upwards along the frequency axis. Indeed, they are lower-bounded by the characteristics of their UFLS schemes (recall that the minima on Fig. 3 derive from the UFLS steps in a relatively straightforward manner), which prevents them from being shifted downwards; but they may reach high values of frequency, potentially above 
TABLE III

SCHEMES COMPARISON

\begin{tabular}{lll}
\hline & Scheme A & Scheme B \\
\hline Maximum load shed & $\begin{array}{l}60 \% \text { - allow larger } \\
\text { imbalances }\end{array}$ & \\
\hline Maximum frequency & $\begin{array}{l}\text { contained due to reaches higher val- } \\
\text { the significant ues } \\
\text { inaccuracy }\end{array}$ \\
\hline Last frequency step & $\begin{array}{l}\text { 47.5 Hz - risk of gen- } \\
\text { erators tripping for } \\
\text { low frequencies is } \\
\text { higher before shed- } \\
\text { ding the total load }\end{array}$ \\
\hline
\end{tabular}

$50 \mathrm{~Hz}$. We will thus now turn to studying how the maximum frequency of Fig. 3 changes when the main parameters of the simulation are changed for each UFLS scheme.

\section{Sensitivity of the max frequency reached post-shedding}

Fig. 3 shows the maximum frequency reached after loadshedding for various imbalances. In this section, we will focus on the maximum frequency reached for a given scheme, when the initial loss of generation varies. For instance, Fig. 3 shows that for the value of inertia $H$, droop constant $g_{d}$ and load sensitivity $k_{p f}$ that are indicated in Section III, the maximum frequency that could be reached for UFLS Scheme A is about $49.50 \mathrm{~Hz}$, and it is about $50.25 \mathrm{~Hz}$ for Scheme B. Now, how do these two values change when parameters $H, g_{d}$ and $k_{p f}$ vary? Note that the initial imbalance that leads to the maximum frequency may not be the same for different values of these three parameters. In the following figures, we will vary one (and only one at a time) of the three parameters under consideration, and plot the maximum postload-shedding frequency with respect to this parameter. The other two parameters are kept constants; they are set to the values indicated in Section III, namely:

$H=6$ seconds, $g_{d}=5 \% / \mathrm{Hz}, k_{p f}=1 \% / \mathrm{Hz}$.

Note that our model does not capture the phenomenon of generators tripping due to excessive frequency; as a consequence, the following graphs display unrealistic high values of frequency (that would be prevented in practice by the fact that generators would trip). In our studies, these values indicate that we detected a "danger zone": values of parameters $H, g_{d}$ and $k_{p f}$ that could lead to an overshoot of the UFLS scheme under consideration.

1) System inertia: power system inertia, $\mathrm{H}$, is an energy stock provided by rotating machines in the network. The larger this energy stock, the smaller the time derivative of frequency (for a given imbalance); see Equation (1).

Fig. 4 shows the evolution of the maximum frequency reached for the two UFLS schemes previously compared. If inertia is larger, the system response will be long, fewer relays will trip because of frequency measurement inaccuracy, and the final value of frequency will be lower, which is beneficial. Conversely of inertia is low, frequency will evolve fast, many relays will trigger, frequency will reach a potentially high value after load shedding.

Particularly, the effect of inertia is stronger on Scheme A because UFLS steps are larger. Thus, for low values of inertia, Scheme B appears better than Scheme A (the beneficial effect of having many steps dominates), while Scheme A appears better than Scheme B for high values of inertia (the beneficial effect of using less accurate frequency measurements dominates).

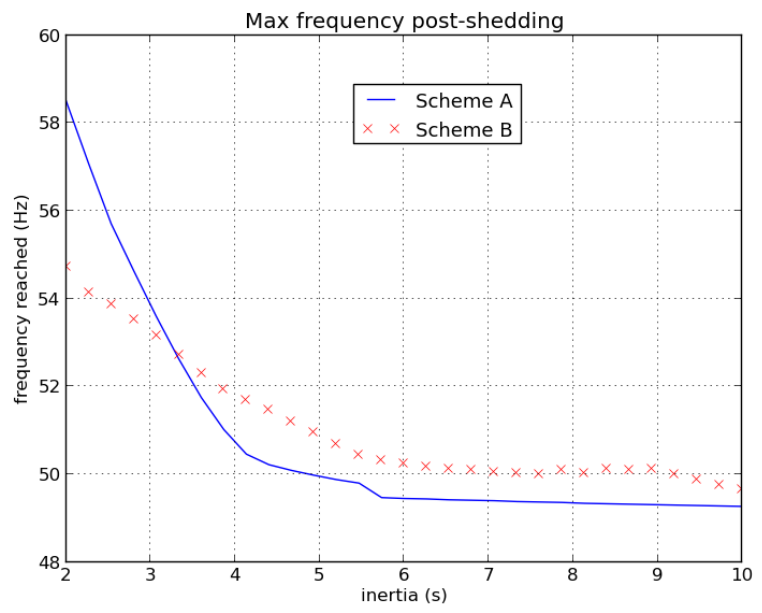

Fig. 4. Evolution of frequency for different values of system inertia, $H$.

2) Generators droop: the generator droop, $g_{d}$, is the primary reserve of the frequency regulation. Interestingly, the qualitative effect of this parameter is not the same for both schemes, see Fig. 5. This may be explained simply by the fact that droop control tends to drive frequency towards (rather than "away from") $50 \mathrm{~Hz}$, as follows. When Scheme A is used, the post-load-shedding frequency is lower than $50 \mathrm{~Hz}$. As a consequence, the effect of droop control when Schema $\mathrm{A}$ is used is to increase post-load-shedding frequency (hence the fact that the curve for Scheme A is nearly monotonically increasing on Fig. 5).

Conversely, when Scheme B is used, the post-load-shedding frequency is higher than $50 \mathrm{~Hz}$, because more accurate relays are used and such relays tend to trigger all-at-once; so the effect of droop control is to decrease frequency (hence the fact that the curve for Scheme A is monotonically decreasing on Fig. 5).

From a certain value of the droop constant, there is no effect on the maximum frequency reached after load shedding (this is particularly visible for Scheme A on Fig. 5). The droop is so important that frequency stability of the system is guaranteed even with imbalances which lead to apply load shedding.

3) Frequency sensitivity of consumption, $k_{p f}$ : on one hand, the primary frequency regulation is almost a proportional gain (limited between $\pm 0.2 \mathrm{~Hz}$ around $50 \mathrm{~Hz}$ ) which allows the system to reach a power balance. On the other hand, the load tends to decrease when frequency drops, which further favors the power balance. 


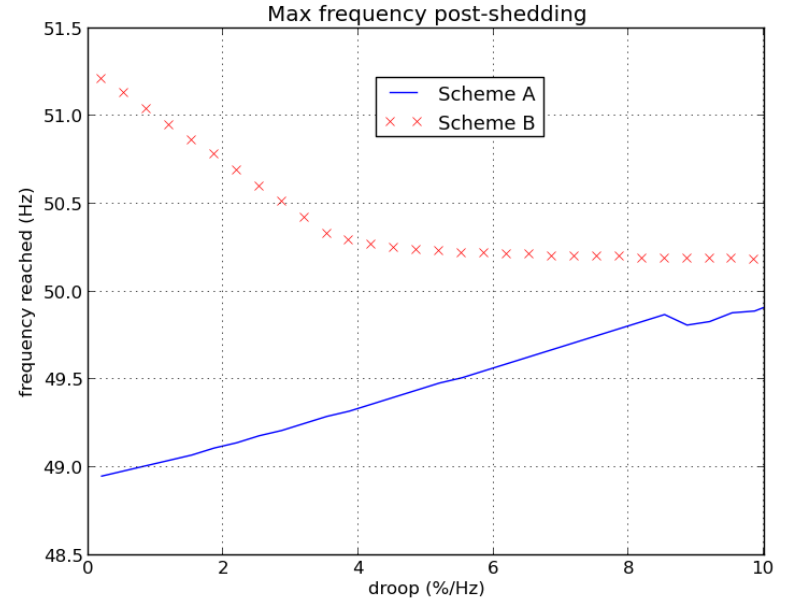

Fig. 5. Evolution of frequency for different generators droop constants, $g_{d}$.

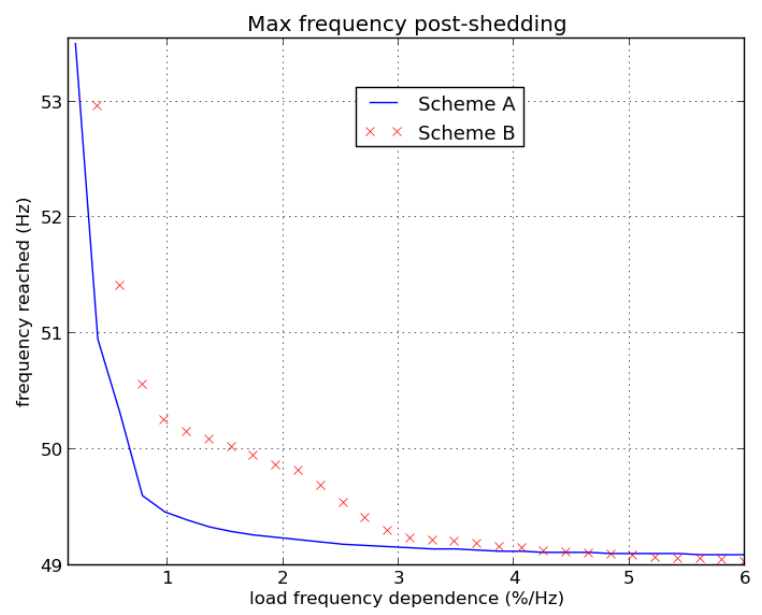

Fig. 6. Evolution of frequency for different values of the sensitivity of load with respect to frequency, $k_{p f}$.

See Fig. 6 for the effect on both schemes of this parameter. The higher $k_{p f}$, the better from the viewpoint of system stability. On the contrary, if load is not sensitive to frequency (or if the sensitivity is even negative), then its effect is detrimental from the viewpoint of system stability. This behavior is the same for both UFLS schemes. Nevertheless the effect if more visible for Scheme A than for Scheme B: the reason is the same that for the inertia.

\section{Conclusions}

The performance criterion defined in Section IV-B allows us to have a basis to compare the two archetypal types of UFLS schemes: the scheme that has been commonly used in the last decades, corresponding to Scheme A, and the future one (yet to be implemented), corresponding to Scheme B. The curve of Fig. 3 representing maximum frequency reached post-shedding in function of the power imbalance looks like saw-teeth for both schemes. Then, whichever scheme is implemented, the frequency reached may correspond to one of the frequency thresholds of the UFLS scheme (the threshold reached will depend on the power imbalance). Thus, it is not possible to design a UFLS scheme in order to regulate the frequency. Moreover, as previously mentioned, an UFLS scheme is the last part of frequency stability and has not a regulation aim but a protection/defense goal of the electrical system.

The sensitivity study depending on grid parameters shows that inertia $H$ and the load frequency dependence $k_{p f}$ have the same beneficial effect on maximum frequency reached for both schemes. Scheme A is however more sensitive than Scheme B to these two parameters, because of its inaccuracy in frequency measurement. For our reference values of $H$ and $k_{p f}$, the droop parameter $g_{d}$ has an opposite effect on the two schemes: when $g_{d}$ is increased, the frequency reached with Scheme A increases towards $50 \mathrm{~Hz}$ while the frequency reached with Scheme B decreases towards $50 \mathrm{~Hz}$. Because of differences in accuracy of frequency measurement, the maximum postshedding frequency reached with Scheme A is relatively low, while Scheme B presents a higher risk of over-frequency situations. This explains why the frequency reached after the shedding that occurred on November 4th, 2006 in Europe was just over $49 \mathrm{~Hz}$. It was due of UFLS schemes of countries involved in shedding which were of the type of the Scheme A developed in this paper.

In the future, the gradual replacement of existing UFLS relays with higher-accuracy ones should be done with care, in order to avoid unintentionally degrading the performance of the UFLS mechanism.

\section{REFERENCES}

[1] Delfino, B., Massucco, S., Morini, A., Scalera, P., \& Silvestro, F. (2001, July). Implementation and comparison of different under frequency loadshedding schemes. In Power Engineering Society Summer Meeting, 2001 (Vol. 1, pp. 307-312). IEEE.

[2] ENTSO-E. Network Code on Emergency and Restoration.

[3] De Boeck, S., \& Van Hertem, D. (2015, June). Under frequency load shedding schemes in systems with high PV penetration: Impact and improvements. In PowerTech, 2015 IEEE Eindhoven (pp. 1-6). IEEE.

[4] Terzija, V. V. (2006). Adaptive underfrequency load shedding based on the magnitude of the disturbance estimation. IEEE Transactions on Power Systems, 21(3), 1260-1266.

[5] Rudez, U., \& Mihalic, R. (2011). Analysis of underfrequency load shedding using a frequency gradient. IEEE transactions on power delivery, 26(2), 565-575.

[6] Xie, J., Liu, C. C., \& Sforna, M. (2015, June). Distributed underfrequency load shedding using a multi-agent system. In PowerTech, 2015 IEEE Eindhoven (pp. 1-6). IEEE.

[7] ENTSO-E. Network Code on Requirements for Grid Connection Applicable to all Generators (RfG).

[8] ENTSO-E. Network Code on Load Frequency Control and Reserves (LFCR).

[9] Concordia, C., \& Ihara, S. (1982). Load representation in power system stability studies. IEEE transactions on power apparatus and systems, 4(PAS-101), 969-977.

[10] Commission de régulation de l'énergie (2007). Rapport d'enquête de la commission de régulation de l'énergie sur la panne d'électricité du samedi 4 novembre 2006.

[11] ENTSO-E. (2014, November). Technical background for the Low Frequency Demand Disconnection requirements.

[12] ENTSO-E. (2015, September). RG CE OH Policy 5: Emergency Operations V 3.0. B G2. 